\title{
Variación de los indicadores de mortalidad evitable entre comunas chilenas como aproximación a las desigualdades de salud
}

\author{
Cesar Gattini, ${ }_{1}^{1}$ Colin Sanderson ${ }^{2}$ y Carlos Castillo-Salgado ${ }^{3}$
}

RESUMEN Objetivos. Analizar variaciones de mortalidad evitable entre comunas, utilizando diversos indicadores, como aproximación operacional para estimar desigualdades de salud.

Métodos. Análisis de variación de áreas pequeñas en una muestra de 117 de las 335 comunas chilenas en 1992. Usando datos secundarios, se desarrollaron y analizaron indicadores de mortalidad evitable, tales como los años de vida potencial perdidos (AVPP), la mortalidad evitable (ME) (con antecedentes y criterios basados en fuentes publicadas), la mortalidad evitable mediante la atención de salud (MEAS), y la esperanza de vida. También se creó un indicador de desarrollo socioeconómico (IDSE). La amplitud de las variaciones observadas entre indicadores se estimó mediante el coeficiente ponderado de variación, el coeficiente de Gini, la razón entre quintiles extremos del IDSE y la razón entre el quintil con el menor IDSE y el grupo de comunas con IDSE mayores de 0,90 (referencia empírica óptima). El perfil socioeconómico de las variaciones se examinó mediante curvas de concentración y la comparación de quintiles comunales según IDSE.

Resultados. Los diversos indicadores de ME usados mostraron una relación inversa estadísticamente significativa con el desarrollo socioeconómico, tendencia también observada en el perfil de los quintiles definidos por IDSE y en la mayoría de las causas específicas de mortalidad evitable. El uso de tres niveles de referencia (promedio, quintil con el mayor IDSE y referencia empírica óptima) plantea la medición de distintas brechas que podrían prevenirse. La razón entre el quintil con el menor IDSE y la referencia óptima fue de 2,1 en el caso de la ME, de 2,0 en el caso de los AVPP, de 1,7 en el de la mortalidad infantil y de 1,5 en el de la MEAS.

Conclusiones. Los resultados, que coinciden con los hallados en otras fuentes publicadas previamente, ponderan la magnitud y el perfil de las variaciones entre comunas y proveen información, basada en datos de 1992, para iniciar un monitoreo de las desigualdades de salud entre áreas geográficas pequeñas, en este caso las comunas. Aunque las iniciativas por mejorar la equidad se concentran en las comunas de menor desarrollo socioeconómico y mayor mortalidad evitable, reducir esta última implica una tarea con un enfoque doble: dar prioridad de intervención a las comunas más postergadas por un lado, y cubrir la mayoría de las comunas para prevenir la mortalidad evitable por el otro.

Palabras clave

Indicadores, mortalidad evitable, desigualdad, Chile.

1 Programa de Organización y Gestión de Servicios de Salud, División de Desarrollo de Sistemas y Servicios de Salud, Organización Panamericana de la Salud. Washington, D.C., EE. UU. Dirección postal: PAHO/WHO, 525 Twenty-third Street, N.W., Washington, D.C. 20037, U.S.A.

2 Health Services Research Unit, Department of Public Health and Policy, London School of Hygiene and Tropical Medicine, University of London, Londres, Reino Unido.
Hay una extensa documentación de la presencia de desigualdades de salud en los distintos países de la Región, aunque

3 Programa Especial de Análisis de Salud, Organización Panamericana de la Salud, Washington, D.C., EE. UU. su naturaleza y amplitud varían $(1,2)$. La mortalidad evitable (ME) se ha utilizado para aproximarse al análisis de las desigualdades de salud, a pesar de que se limita a representar el máximo daño que esta puede sufrir y el extremo final de la vida $(3,4)$. Se considera que una 
muerte es evitable cuando ocurre de forma innecesaria $y$ prematura, $y$ cuando se puede prevenir mediante acciones individuales $\mathrm{o}$ sociales orientadas a controlar factores determinantes del entorno socioeconómico, o mediante la atención de la salud $(5,6)$. Por ello, las variaciones en la frecuencia de las muertes evitables no solo reflejan desigualdades de salud, sino que también apuntan a la posible necesidad de emprender intervenciones sanitarias. A su vez, por "necesidad de salud" se ha de entender la brecha entre un estado observado y uno deseado (normativo o empírico) (7).

Diferentes tipos de indicadores relacionados con la mortalidad han sido ampliamente empleados para analizar desigualdades evitables en el área de la salud. Algunos de ellos son la mortalidad ajustada, la esperanza de vida, los años de vida potencial perdidos (AVPP) y la ME (8-10). A escala colectiva, la evitabilidad puede entenderse desde el punto de vista operacional en relación con los niveles de salud que es factible alcanzar, según estimaciones teóricas (basadas en la opinión de expertos y en los conocimientos adquiridos) o con niveles deseables ya alcanzados en poblaciones que sirven a manera de referencia óptima (empírica) (11-13).

La premisa básica que encierra el indicador AVPP es que una muerte evitable es prematura en la medida en que, de haberse prevenido, una persona hubiese vivido tanto como el resto de la población, es decir, hasta una edad fijada de forma convencional, que en este estudio es de 65 años.

Entre los métodos notificados en fuentes bibliográficas para analizar cómo varían los indicadores entre distintas áreas geográficas o grupos de interés se destacan los siguientes: la descripción del comportamiento de las variables y sus relaciones estadísticas; el uso del coeficiente de Gini, del coeficiente de concentración y de las curvas de Lorenz y de concentración; la comparación entre quintiles $\mathrm{u}$ otras agrupaciones de la población; y el análisis del perfil de las variaciones socioeconómicas, además de otros métodos de comparación más complejos $(4,14,15)$.
Hay limitaciones conceptuales y metodológicas propias de este tipo de estudio que emanan de los criterios utilizados (como la evitabilidad o prematurez de la muerte), el modo de desarrollar los indicadores, o los problemas que surgen al comparar áreas geográficas distintas $(11,16)$. Sin embargo, estos estudios constituyen una respuesta posible y práctica para analizar del modo más detallado posible las variaciones a escala local, en particular si no se cuenta con otras formas de investigar $y$ hay necesidades de información y análisis para orientar políticas tendientes a disminuir las desigualdades entre distintas áreas y grupos de la población, sobre todo cuando hay indicios de que estas desigualdades tienden a persistir en los países de las Américas $(2,17)$.

\section{MATERIALES Y MÉTODOS}

Se analizaron las variaciones observadas entre áreas geográficas pequeñas (comunas). La muestra se compuso de 117 de las 335 comunas que había en Chile en 1992 (es decir, de 41,1\% de las comunas del país, donde vivía 77,3\% de la población). La unidad de análisis fue la comuna; la población de cada comuna varió de menos de mil habitantes hasta más de trescientos mil, con un promedio de 42000 habitantes. Se obtuvieron datos secundarios de diversas fuentes nacionales: la población se obtuvo del Censo Nacional de Vivienda y Población de 1992 (18); las defunciones registradas en 1990-1994 fueron proporcionadas por el Ministerio de Salud (se incluyeron todas las de este período debido al bajo número de defunciones por causas específicas en muchas comunas); los datos socioeconómicos provinieron del Instituto Nacional de Estadísticas (INE) y la Encuesta de Hogares Casen, 1992 (Ministerio de Planificación Social, MIDEPLAN) (19).

Se desarrollaron y compararon varios indicadores: AVPP, ME (según antecedentes y criterios basados en fuentes publicadas), mortalidad evitable mediante la atención de la salud (MEAS), esperanza de vida y un indicador de desarrollo socioeconómico (IDSE).

Para calcular los AVPP se tomó la diferencia entre la edad de cada persona que murió y la edad de referencia base (65 años). Posteriormente se calculó la razón dada por los AVPP por cada 1000 habitantes.

La estimación de la tasa de mortalidad evitable (ME) se basó en una lista de 29 causas o grupos de causas y edades, según las fuentes bibliográficas con criterios y listas usadas previamente en Chile (20-25). Las causas específicas, las edades y el código de la Clasificación internacional de enfermedades, novena revisión (CIE-9) se describen en el cuadro 1. A partir de la lista de causas se desarrolló un indicador más específico - la MEAS - en el que se incorporaron cinco grupos de trastornos que se asocian con una mortalidad que puede prevenirse mediante la atención médica: leucemia; insuficiencia renal crónica; hernia abdominal, apendicitis, peritonitis y obstrucción intestinal; traumatismo de cráneo; y traumatismo interno de tórax, abdomen y pelvis.

El índice de desarrollo socioeconómico se estimó usando el método empleado por el Programa de Desarrollo de las Naciones Unidas para calcular el Índice de Desarrollo Humano (IDH) (26), que toma en consideración la ubicación del valor de un indicador en cada comuna en relación con los valores máximo y mínimo del intervalo dado por los valores de todas las comunas. La siguiente fórmula fue aplicada para estandarizar cada variable:

Valor de la variable $=$ (valor observado valor mínimo del intervalo) / (valor máximo del intervalo - valor mínimo)

Para cada comuna, el IDSE se calculó sumando los valores de cada componente:

\section{IDSE comunal $=[(0,53$ pobreza $)+$ $(0,53(\log )$ ingreso $)+$ años de escuela + alcantarillado] / 3}

La selección de los componentes, basada en los que se utilizan para el IDH, fue discrecional: no se contemplaron ni la mortalidad infantil ni la esperanza de 
CUADRO 1. Distribución de causas específicas de mortalidad evitable por quintiles socioeconómicos según el índice de desarrollo social y económico (IDSE) en comunas chilenas, 1992

\begin{tabular}{|c|c|c|c|c|c|c|c|c|c|c|c|}
\hline $\begin{array}{l}\text { Código } \\
\text { CIE 9b }\end{array}$ & Causa de muerte & $\begin{array}{c}\text { Edad } \\
\text { (años) }\end{array}$ & \multicolumn{5}{|c|}{ Quintil de IDSE (1) } & $\begin{array}{c}\text { REO } \\
(2)\end{array}$ & \multicolumn{2}{|c|}{ Razón } & $\begin{array}{l}I^{a} \\
\text { (3) }\end{array}$ \\
\hline 001-139 & Enfermedades infecciosas & $0-64$ & 9,0 & 7,2 & 7,8 & 8,5 & 5,2 & 3,7 & 1,74 & 2,42 & $-0,078$ \\
\hline 174 & Cáncer de mama en mujeres & $35-64$ & 19,7 & 21,4 & 20,5 & 22,4 & 28,0 & 24,6 & 0,71 & 0,80 & 0,073 \\
\hline 180 & Cáncer cervicouterino & $35-64$ & 29,7 & 19,8 & 25,8 & 20,9 & 12,9 & 5,3 & 2,30 & 5,64 & $-0,130$ \\
\hline 250 & Diabetes mellitus & $35-64$ & 11,4 & 13,7 & 12,5 & 12,1 & 8,9 & 4,6 & 1,29 & 2,47 & $-0,049$ \\
\hline $390-459$ & Enfermedades aparato circulatorio & $35-64$ & 126,4 & 113,8 & 107,4 & 116,6 & 89,4 & 67,9 & 1,41 & 1,86 & $-0,057$ \\
\hline 393-398 & Fiebre reumática & $35-64$ & 4,6 & 5,7 & 4,6 & 4,1 & 4,1 & 3,1 & 1,13 & 1,52 & $-0,047$ \\
\hline $410-414$ & Enfermedad isquémica corazón & $35-64$ & 43,5 & 38,8 & 34,3 & 43,1 & 37,0 & 33,3 & 1,18 & 1,31 & $-0,021$ \\
\hline $430-438$ & Enfermedad cerebrovascular & $35-64$ & 50,4 & 39,3 & 41,2 & 40,0 & 28,5 & 19,0 & 1,77 & 2,65 & $-0,021$ \\
\hline $460-519$ & Enfermedades del árbol respiratorio & $0-64$ & 19,3 & 20,7 & 18,6 & 19,2 & 12,8 & 8,6 & 1,51 & 2,26 & $-0,068$ \\
\hline 571 & Cirrosis hepática & $35-64$ & 59,7 & 61,1 & 64,4 & 50,6 & 33,5 & 13,4 & 1,78 & 4,44 & $-0,095$ \\
\hline $574-576$ & Colelitiasis & $35-64$ & 2,5 & 3,0 & 2,9 & 3,2 & 1,7 & 0,4 & 1,46 & 5,72 & $-0,056$ \\
\hline 585 & Insuficiencia renal crónica & $15-64$ & 2,4 & 2,1 & 1,5 & 2,2 & 1,4 & 0,8 & 1,66 & 3,00 & $-0,095$ \\
\hline 590 & Infecciones renales & $35-64$ & 0,9 & 0,8 & 1,1 & 1,1 & 0,9 & 0,1 & 1,03 & 6,85 & 0,011 \\
\hline $630-676$ & Causas obstétricas & $15-44$ & 3,3 & 3,1 & 2,5 & 2,9 & 1,8 & 1,2 & 1,88 & 2,74 & $-0,114$ \\
\hline 630-639 & Aborto & $15-44$ & 1,0 & 0,9 & 0,8 & 0,8 & 0,4 & 0,6 & 2,24 & 1,57 & $-0,141$ \\
\hline $640-676$ & Otras causas obstétricas (no el aborto) & $15-44$ & 2,4 & 2,1 & 1,7 & 2,1 & 1,3 & 0,6 & 1,76 & 3,96 & $-0,103$ \\
\hline 001-999 & Muertes neonatales & 0 & 7,8 & 7,0 & 7,3 & 6,5 & 5,3 & 5,0 & 1,47 & 1,57 & $-0,070$ \\
\hline 001-999 & Muertes infantiles tardías & 0 & 7,0 & 6,4 & 6,3 & 5,2 & 4,1 & 3,7 & 1,71 & 1,88 & $-0,107$ \\
\hline 001-999 & Muertes infantiles tardías & 0 & 14,8 & 13,5 & 13,6 & 11,8 & 9,4 & 8,7 & 1,57 & 1,70 & $-0,087$ \\
\hline
\end{tabular}

a IC: Índice de concentración.

${ }^{6}$ CIE-9: Clasificación internacional de enfermedades, novena revisión.

${ }^{c}$ REO: Referencia empírica óptima.

vida, ya que son variables relacionadas con la salud. En su lugar se incluyó la existencia de alcantarillado. Se tomó la expresión logarítmica del ingreso, dada la gran dispersión de los ingresos entre las comunas. En el caso de la pobreza - un factor de desarrollo negativo- se utilizó el valor invertido (es decir, se asignó 1,0 al grado mínimo y 0,0 al máximo). La validez del índice se estimó posteriormente mediante la comparación con índices chilenos similares utilizados con anterioridad: el IDH (26), la "vulnerabilidad infantil" (índice compuesto a partir de factores de riesgo infantil, desarrollado por Mardones et al. [27]), y el índice de prioridad social (28). El IDSE muestra una correlación estadística altamente significativa con esos otros índices.

Para medir la amplitud de las variaciones entre las comunas se emplearon como medidas resumen ${ }^{4}$ el coeficiente ponderado de variación, el

4 En ocasiones denominadas "medidas sintéticas" en español y conocidas en inglés por summary measures. coeficiente de Gini y la razón entre quintiles extremos; se examinaron las relaciones entre las variaciones observadas entre las comunas para cada indicador de salud y de desarrollo socioeconómico; y se examinó el perfil de las variaciones entre los quintiles con diversos IDSE, el cual se comparó con el del promedio ponderado, el del quintil con el IDSE más alto y el del grupo de comunas de mayor desarrollo (referencia empírica óptima).

El coeficiente ponderado de variación (CPV) se estimó mediante la fórmula siguiente: 
$C P V=$ (desviación estándar ponderada $)$ promedio ponderado)

La ponderación se hizo de acuerdo con la población de cada comuna. El coeficiente de Gini se estimó mediante la siguiente fórmula:

$C G=1-\operatorname{Sum}\left(Y_{\mathrm{i}+1}+Y_{i}\right)\left(X_{i+1}-X_{\mathrm{i}}\right)$

siendo $X_{i}$ la proporción acumulada de la población (comunas) ordenada por niveles de acuerdo con el indicador de interés (por ejemplo, los AVPP), y $Y_{i}$ la proporción acumulada de los acontecimientos en que se basa ese indicador. La curva de Lorenz se basó en los valores $x$ y y de cada comuna.

El índice de concentración (IC) y su correspondiente curva son similares al coeficiente de Gini y a la curva de Lorenz, pero se basan en la proporción acumulativa de acontecimientos o muertes en comunas ordenadas según su grado de desarrollo socioeconómico (y no según el propio indicador de interés).

El perfil de las variaciones se describió comparando quintiles ordenados por el IDSE; el tamaño de estos últimos representa la cantidad de población agrupada y no el número de comunas. El promedio ponderado de los valores de las comunas fue tomado como el valor resumen para cada quintil.

El promedio ponderado de las tasas de mortalidad observadas en las comunas más privilegiadas económicamente (con un IDSE mayor de 0,90) constituyó la referencia empírica óptima (REO). Este grupo, integrado por cuatro comunas, se ubica en el sector oriental de la capital y tiene el nivel de ingresos y de vida más alto del país. Prácticamente no tiene pobreza y su tasa de mortalidad es muy baja (claramente menor que la del quintil con el IDSE más alto).

\section{RESULTADOS}

El IDSE muestra una correlación inversa y estadísticamente significativa con los AVPP $(r=-0,661 ; P<0,001)$, ME $(r=-0,393 ; P<0,001)$ y la mortalidad infantil $(r=-0,431 ; P<0,001)$, aunque no se observó ninguna correlación significativa con la MEAS.

Como primera aproximación a la relación entre las desigualdades sociales y la mortalidad evitable, en la figura 1 se muestra, para cada comuna, la relación entre los valores de AVPP e IDSE y entre los valores de ME e IDSE. Al examinarse la tendencia hacia una relación inversa entre los AVPP y el IDSE, se observa que las variaciones de los AVPP muestran una mayor dispersión entre comunas cuyos IDSE son similares y bajos $(<0,20)$. Esto podría obedecer a distintas razones: el azar, el tamaño reducido de las comunas - y por lo tanto la escasez de muertes-, o factores distintos a los captados en el IDSE (p. ej., la cercanía a comunas con un mayor desarrollo o con mayores posibilidades de tener acceso a la atención de salud). Al examinar cada comuna individualmente, se destacó que en las nuevas comunas constituidas por la clase media (nuevos polos de desarrollo), hay menos AVPP que en las que siempre fueron pobladas por dicha clase, aunque en ambos casos los IDSE sean similares. Los valores de AVPP del grupo de comunas con un IDSE > 0,90 son bastante bajos, invariablemente se ubican por debajo de la línea de tendencia, lo que sugiere que a partir de cierto nivel de desarrollo se puede acentuar la tendencia a que haya menos muertes prematuras. La figura que compara la ME y el IDSE se parece a la anterior, pero en general muestra más dispersión en torno a la línea de tendencia que ilustra la relación inversa entre la ME y el IDSE, lo cual es compatible con una menor correlación. Es probable que en esto influyan las

FIGURA 1. Años de vida potencial perdidos (AVPP) (por 1000 habitantes) frente a índice de desarrollo socioeconómico (IDSE), y muertes evitables (ME) (por 1000 habitantes) frente a IDSE en comunas chilenas, 1992
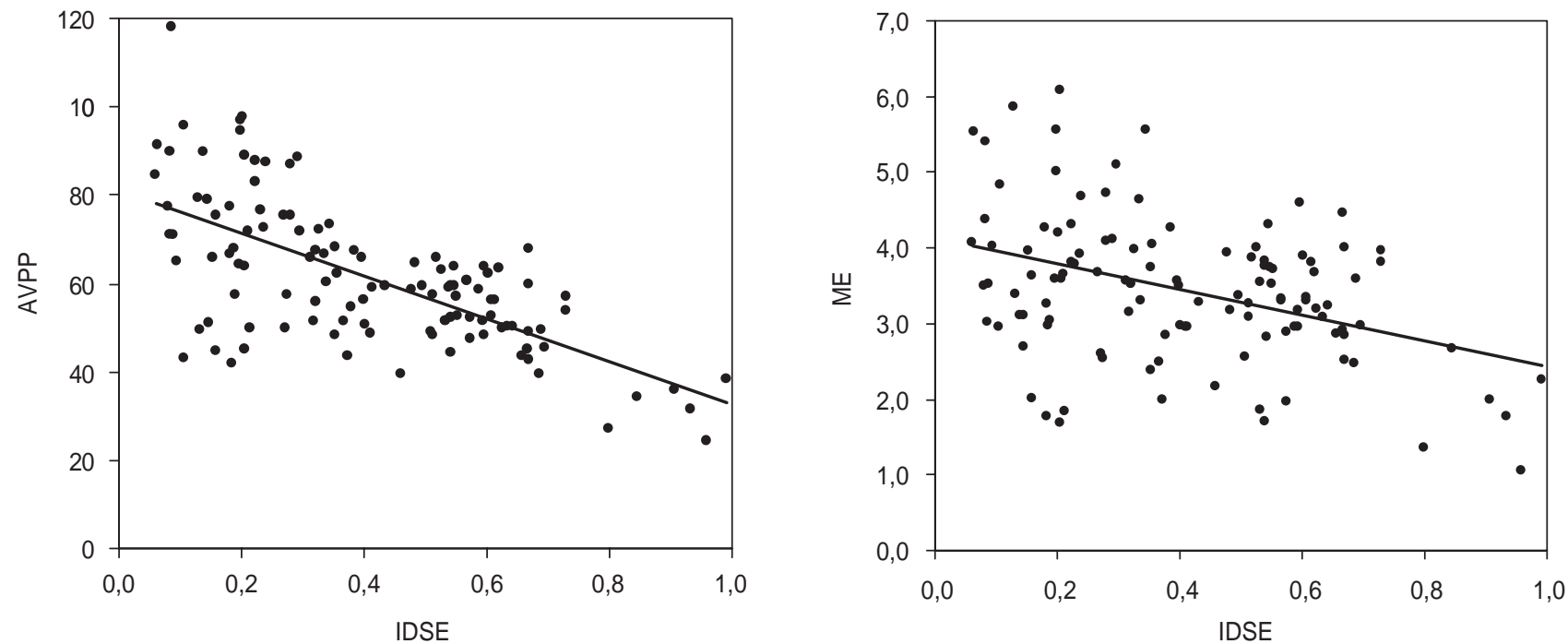
CUADRO 2. Medidas que resumen las variaciones de distintos indicadores de mortalidad evitable en comunas chilenas, 1992

\begin{tabular}{lcrrrrrrr}
\hline \multicolumn{1}{c}{ Indicador } & $\begin{array}{c}\text { Promedio } \\
\text { ponderado }\end{array}$ & Asimetría & Q1 & Q5 & $\begin{array}{r}\text { Razón } \\
\text { Q5/Q1 }\end{array}$ & CPV & $\begin{array}{r}\text { Coeficiente } \\
\text { de Gini }\end{array}$ & $\begin{array}{c}\text { Correlación } \\
\text { con AVPP }\end{array}$ \\
Valor $P$
\end{tabular}

a Por 1000 habitantes.

b Por 1000 nacidos vivos.

c En inglés skewness.

Nota: TMA: tasa de mortalidad ajustada; AVPP: años de vida potencial perdidos; CPV: coeficiente ponderado de variación.

pocas muertes específicas que pueden ocurrir en comunas pequeñas.

Además de la variabilidad de los indicadores de ME desarrollados, se examinó también la de otros indicadores candidatos, como las tasas de mortalidad ajustada total y de la población de 0 a 64 años, y la mortalidad bruta en distintos grupos de edad (cuadro 2). Se destaca que todos los indicadores variaron notablemente según las diversas medidas de variación (con la excepción de la esperanza de vida) y mostraron una correlación estadística significativa con los AVPP.

La figura 2 muestra las curvas de concentración de ME y MEAS de la población de las comunas agrupadas según su IDSE. Ambas evidencian desigualdades en los valores de los indicadores, particularmente en los de la ME. Sin embargo, las desigualdades de la $\mathrm{ME}$ se ven gráficamente menores que las observadas en la figura 1 debido a que las comunas con menor IDSE y mayor ME tienden a tener poca población y por ello ejercen poco peso como factor de alejamiento (desigualdad) respecto de la línea de igualdad.

El perfil de distribución de los indicadores de mortalidad en las comunas agrupadas en quintiles según el IDSE se muestra en la figura 3. Estas variaciones están expresadas en relación con el valor promedio ponderado de las comunas (referencia: 1,0). El perfil de variación según quintiles guarda una proporción inversa con el desarrollo socioeconómico, que incluso tiende a descender aun más entre los quintiles 4 y 5 , que son los que muestran el mayor IDSE. En la figura 3, el nivel total de cada quintil presenta cuatro posibles

FIGURA 2. Curvas de concentración de la mortalidad evitable (ME) y de la mortalidad evitable mediante la atención de la salud (MEAS) en la población acumulada, por comunas ordenadas según su índice de desarrollo social y económico (IDSE). Chile, 1992
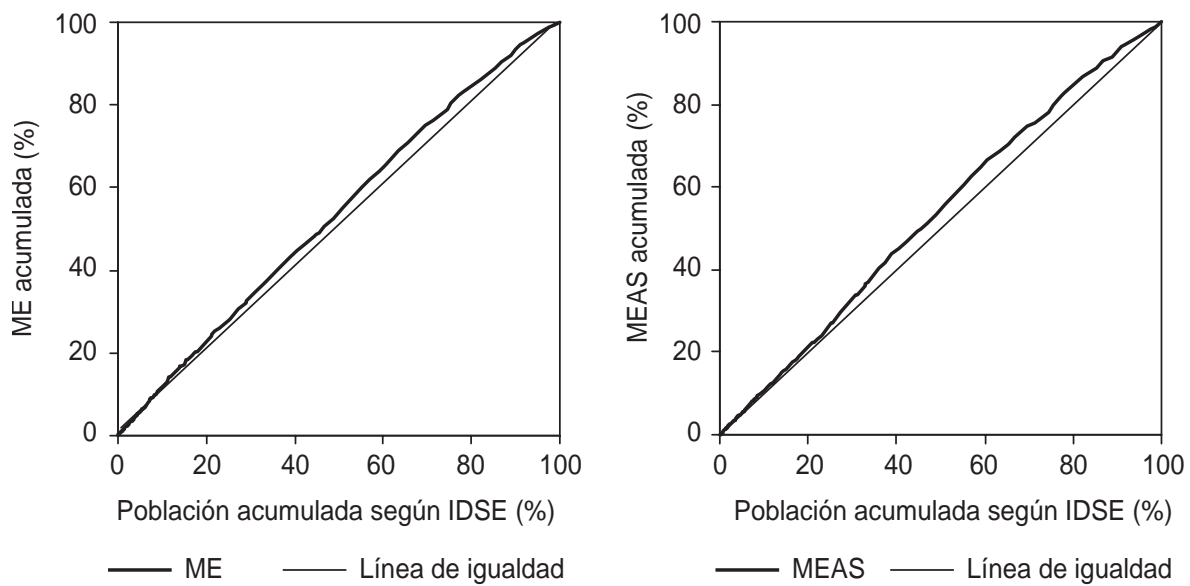
FIGURA 3. Distribución de indicadores de mortalidad evitable (ME) por quintiles socioeconómicos, según el índice de desarrollo social y económico (IDSE) en comunas chilenas, con intervalos de evitabilidad empírica, 1992
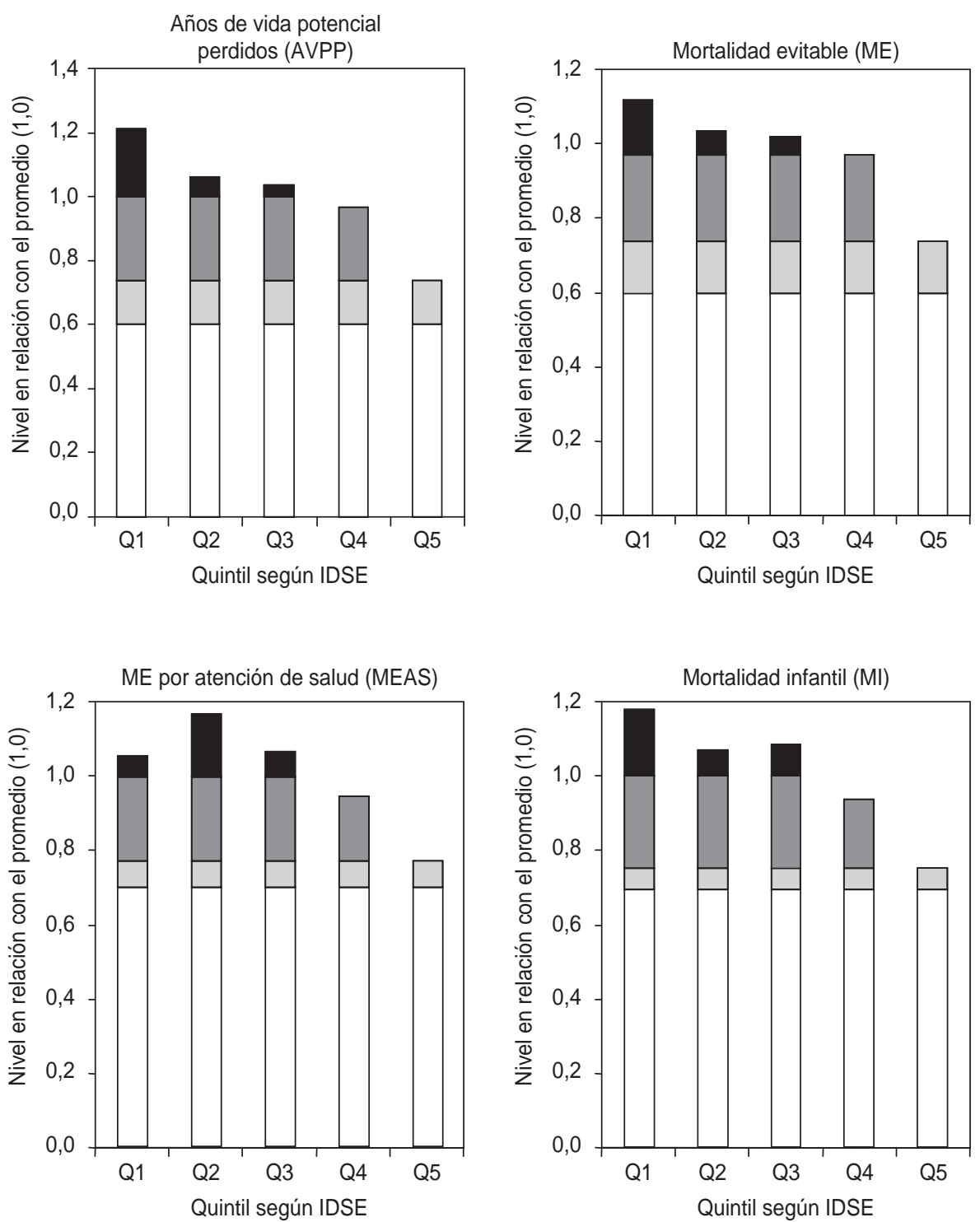

Intervalo de evitabilidad: $\square$ intervalo por debajo de la referencia empírica óptima (REO); $\square$ intervalo entre la referencia empírica óptima y el quintil 5 (Q5); $\square$ intervalo entre el Q5 y el promedio comunal ponderado; $\square$ intervalo por encima del promedio comunal ponderado.

intervalos de referencia empírica en relación con la evitabilidad de la muerte, tomando en cuenta los tres niveles de referencia: el promedio ponderado de las comunas (valor 1,0), el mejor quintil del IDSE y la REO. En cada quintil se muestra: a) un recorrido bajo de la REO, que puede considerarse inevitable en el contexto chileno en el momento del estudio (ni siquiera se redujo en las comunas más privilegiadas); b) un intervalo entre la REO y el quintil de mayor IDSE; c) un intervalo entre el quintil de mayor IDSE y el promedio ponderado de las comunas; y d) un intervalo entre el promedio ponderado de las comunas y el nivel actual del quintil. Este último intervalo en general se aprecia para los tres quintiles de menor IDSE. La razón entre el quintil con el menor IDSE y la referencia óptima es de 2,1 en el caso de la ME, de 2,0 en el de los AVPP, de 1,7 en el de la mortalidad infantil y de 1,5 en el de la MEAS.

La mayoría de las causas específicas de mortalidad evitable también tienen un comportamiento similar al de los indicadores integrados de AVPP o ME (cuadro 1).

\section{DISCUSIÓN}

Estos resultados coinciden con los notificados en otros trabajos publicados acerca de la relación entre el desarrollo socioeconómico y la salud, resumen la extensión y distribución del tipo de desigualdades estudiadas, y muestran un perfil de variaciones que tiende a disminuir a medida que aumenta el desarrollo socioeconómico. Estudios que han usado indicadores socioeconómicos compuestos, como el índice de privación (en inglés, deprivation index) empleado por Townsend et al. (3) o el de Carstair y Morris (16) para analizar diferencias de salud, también indican que la ME (indicadores de mortalidad general estandarizada o por causas evitables específicas, como el cáncer de cuello uterino, la neumonía y demás) es mayor en las áreas geográficas con mayor privación socioeconómica (16). En Chile, el indicador compuesto de vulnerabilidad infantil en el nivel de la comuna también evidencia la asociación inversa entre los componentes específicos de los indicadores socioeconómicos y la mortalidad, aunque solo se remite a la infantil (27). Al comparar las distintas regiones de Chile, Solís et al. (24) encontraron una asociación persistentemente inversa entre las distintas causas de mortalidad (general y por causas específicas, incluidas algunas causas de mortalidad evitable) e indicadores socioeconómicos específicos (tales como educación, ocupación e urbanización), observándose que la mortalidad evitable se concentra en lugares donde hay mayor privación (24). 
La ME y los AVPP, como indicadores, muestran una correlación significativa entre sí y con el desarrollo socioeconómico, lo cual a su vez confiere validez interna a estos indicadores, que presentan variaciones $y$ comportamientos similares en relación con las diferencias socioeconómicas entre comunas (cuadro 2). El comportamiento estadístico de la MEAS, como indicador, no permite apoyar su uso como marcador, pues no mostró correlación significativa con el desarrollo socioeconómico, quizá debido al escaso número de causas y muertes incluidas o a que sus variaciones dependen de otros factores que no siempre se relacionan con el desarrollo socioeconómico (como el acceso a una buena atención de salud). La mayoría de las causas específicas de ME también tienen un comportamiento similar a la de los indicadores integrados de AVPP o ME (cuadro 1).

El uso de un reducido conjunto de indicadores también provee una visión más amplia y coherente del perfil de las desigualdades en los daños de salud evitables, las cuales a su vez reflejan diferentes necesidades en materia de salud.

La limitación de este tipo de estudio estriba principalmente en la calidad y cobertura de la información utilizada $\mathrm{y}$ en las premisas aplicadas al elegir aquellas causas que se consideran evitables. De ahí que la definición de evitabilidad sea principalmente operacional o normativa. La relación entre el desarrollo socioeconómico y el daño de salud evitable refleja no solo una influencia directa de factores socioeconómicos que ponen en riesgo la salud, sino además la influencia de los distintos grados de atención de salud. Esta última, a su vez, se relaciona directamente con el desarrollo socioeconómico, lo cual explica que haya menos recursos sanitarios y menos atención de salud en lugares con un desarrollo socioeconómico menor, y que en ellos también haya más daños de salud evitables (29).

Tratar de reducir la ME de acuerdo con su distribución socioeconómica implica un doble ámbito de intervenciones del sector de la salud: unas enfocadas preferentemente hacia las comunas más postergadas (estableciendo prioridades tendientes a favorecer la equidad) y otra más amplia, dirigida a la mayoría de las comunas, encaminada a prevenir y posiblemente reducir la mortalidad evitable hasta alcanzar niveles similares a los ya logrados en las comunas más privilegiadas en términos sociales. Ambos tipos de acción requieren estrategias complementarias, especialmente en torno a un enfoque preventivo amplio.

El análisis socioeconómico de la ME puede enriquecer el análisis de la situación de salud a escala local con la información recolectada rutinariamente, que a su vez puede ser un instrumento importante para quienes toman las decisiones, particularmente a la luz de la necesidad de monitorear y evaluar procesos tales como la reforma sectorial, la descentralización de los servicios, y las brechas observadas localmente en las necesidades de salud insatisfechas. Las causas específicas de la ME pueden dar pie a acciones más concretas en forma de distintos tipos de programas o intervenciones de salud, como serían, por ejemplo, los encaminados a combatir la mortalidad infantil y a fomentar la atención prenatal y del parto.

Los resultados expuestos, que coinciden con los hallados en otros trabajos publicados, ponderan la magnitud y el perfil de las variaciones entre comunas y proveen información basada en los datos de 1992 para el seguimiento de desigualdades sociales en el ámbito de la salud que pueden reducirse mediante intervenciones enfocadas en diversos factores, ya sean individuales, del contexto socioeconómico o de la atención de salud.

\section{REFERENCIAS}

1. Benzeval M, Judge $K$, Whitehead $M$, eds. Tackling inequalities in health: an agenda for action. London: Kings's Fund; 1995.

2. Pan American Health Organization. Chapter 1. Health situation analysis En: Annual Report of the Director. The search for equity. Washington, D.C.: PAHO; 1995. Pp. 1-11.

3. Townsend P, Davidson N, Whitehead M. Inequalities in health: the black report and the health divide. London: Penguin; 1988.

4. Braveman P. Monitoring equity in health: a policy-oriented approach in low- and middleincome countries. Geneva: WHO; 1998. (Documento WHO/CSH/HSS/98.12).

5. Whitehead M. The concepts and principles of equity and health. Int J Health Serv 1992;22: 429-445.

6. World Health Organization. Equity in health and health care: a WHO/SIDA initiative. Geneva: WHO/ARA; 1996. (WHO/ARA/96.1).
7. Liss PE. Health care need: meaning and measurement. Hants: Avebury Ashgate Publishing, Ltd.; 1993.

8. Palloni A. Review of data sources and methods for the assessment of trends, age patterns and differentials of mortality in the Third World. En: Cleland J, Hill A, eds. The health transition: methods and measures. Proceedings of an International Workshop, London, June 1989. Canberra: The Australian National University; 1991. Pp. 13-33. (Health Transitions Series No. 3).

9. López A. Causes of death in industrial and developing countries: Estimates for 19851990. En: Jamison D, Mosley H, Measham A, Bobadilla JL, eds. Disease control priorities in developing countries. New York: Oxford Medical Publications; 1993. Pp. 35-50.

10. Dyson T, Murphy M. Macro-level study of socio-economic development and mortality: adequacy of indicators and methods of statistical analysis. En: Cleland J, Hill A, eds. The health transition: methods and measure. Proceedings of an International Workshop, London, June 1989. Canberra: The Australian National University; 1991. Pp. 147-164. (Health Transitions Series No. 3).

11. Figueras J. Effective health care planning. The role of financial allocation mechanisms. [Tesis de doctorado]. Department of Public Health and Policy LSHTM. University of London; 1993.

12. Jougla E, Ducimetiere P, Bouvier Colle $\mathrm{MH}$, Hatton F. Relation between the level of the development of the care system and the level of "preventable" mortality according to department in France. Rev Epidemiol Sante Publique 1987;35:365-377.

13. Murray CJL, Yang G, Qiao X. Adult mortality: levels, patterns and causes. En: Feachem R, 
Kjellstrom T, Murray CJL, Over M, Phillips M, eds. Adult health in the population of the developing world. Oxford: Oxford University Press for the World Bank; 1993. Pp. 135-188

14. Kunt A, Mackenbach J. Measuring socioeconomic inequalities in health. Copenhagen: WHO Regional Office for Europe; 1997. (Discussion Paper EUR/ICP/RPD 416).

15. Organización Panamericana de la Salud. El Progreso de la salud de la población. Informe anual del Director 2000. Washington D.C.: OPS; 2000.

16. Carstairs V, Morris R. Deprivation and health in Scotland. Aberdeen: Aberdeen University Press; 1991

17. Schneider MC, Castillo-Salgado C, LoyolaElizondo E, Bacallao J, Mujica O, Vidaurre O, Alleyne G. Trends in infant mortality inequalities in the Americas: 1955-1975. J Epidemiol Community Health 2002;56:538-541.

18. Instituto Nacional de Estadísticas. Censo nacional de población y vivienda en Chile, 1992. Santiago, Chile: INE; 1993.
19. Ministerio de Planificación Social. Estudio de caracterización socioeconómica nacional, 1992. Santiago, Chile: MIDEPLAN; 1994.

20. Rutstein DD, Berenberg W, Chalmers TC, Fishmen AP, Perrin EB. Measuring the quality of medical care. New Engl J Med 1976;294:582-588.

21. Charlton J, Velez R. Some international comparisons of mortality amenable to medical intervention. BMJ 1986;292:295-301.

22. Holland WW. European atlas of avoidable deaths. 2nd ed. Oxford: Oxford Medical Publications; 1994. (Commission of the HSR. Series No. 6;1).

23. Organización Panamericana de la Salud. Mortalidad evitable: indicador o meta? Aplicación en países en desarrollo. Bol Org Panam Salud 1990;11:1-14.

24. Solis F, Mardones G, Castillo B. Calidad de la atención médica en los servicios de salud del país. Informe final de investigación CONYCIT y Escuela de Salud Pública de Universidad de Chile. Santiago, Chile: Facultad de Medicina, Universidad de Chile; 1992
25. Chile, Ministerio de Salud. Estudio carga de enfermedad. Informe final. Santiago, Chile: MINSA; 1996

26. Programa de las Naciones Unidas para el Desarrollo. Desarrollo humano en Chile, 1996. Santiago, Chile: PNUD; 1996.

27. Mardones Restat F, Díaz M, Bonnefoy JC. Una propuesta de clasificación de las comunas del país, según la situación de la infancia. Santiago, Chile: UNICEF; 1994.

28. Bonnefoy JC. Prioridad social en comunas chilenas. Informe borrador. Santiago: MIDEPLAN; 1995.

29. Hart JT. The inverse care law. Lancet 1971;1: $405-412$

Manuscrito recibido el 19 de julio de 2002. Aceptado para publicación, tras revisión, el 4 de octubre de 2002

ABSTRACT Objectives. To analyze differences in avoidable mortality among communes in Chile, using different indicators as an operational approach to estimating health inequalities. Methods. Small area variation analysis in a sample of 117 of all 335 Chilean

Using different indicators of preventable mortality as an approach to measuring health inequalities in Chilean municipalities communes that existed in 1992. By using secondary data, we developed and compared some avoidable-mortality indicators, such as potential years of life lost (PYLL), avoidable mortality (AM) (based on background and criteria drawn from the literature), health care avoidable mortality (HCAMR), and life expectancy. A socioeconomic development index (SEDI) was also developed. The scope of the variation was estimated through the weighted variation coefficient, the Gini coefficient, the ratio between the values for the quintiles at both extremes of the SEDI distribution, and the ratio of the lowest SEDI quintile to the group of municipalities having a SEDI greater than 0.90 (optimal empirical reference value). The socioeconomic pattern of variations was examined through concentration curves and by comparing communal quintiles based on their SEDI.

Results. The various avoidable-mortality indicators used showed an inverse and statistically significant correlation with socioeconomic development, as well as with the profile of the various SEDI quintiles and with the majority of specific causes of avoidable mortality. The distribution profile of AM indicators among SEDI communal quintiles reflects the same tendency, along with most of the mortality from specific avoidable causes. The use of three reference values (the mean, the quintile with the greatest SEDI, and the optimal empirical reference value) makes it possible to measure gaps that could be avoided. The ratio of the lowest SEDI quintile to the empirical optimal reference value was 2.1 for AM, 2.0 for PYLL, 1.7 for infant mortality, and 1.5 for HCAMR.

Conclusions. These results, which are consistent with those found in previous published sources, estimate the magnitude and pattern of variations among communes. The results also provide information, based on data for 1992, with which to start monitoring health inequalities among small geographic areas, which were communes in this particular case. Although interventions for promoting equity tend to focus exclusively on communes having lower socioeconomic development and higher rates of avoidable mortality, reducing the latter implies a two-pronged approach: prioritizing interventions targeting underprivileged communes so as to foster equity, while attempting to cover the majority of communes in an effort to prevent avoidable mortality. 